Research Paper

\title{
Temporomandibular Disorders in Psoriasis Patients with and without Psoriatic Arthritis: An Observational Study
}

\author{
Vito Crincoli $^{\bowtie}$, Mariasevera Di Comite ${ }^{2}$, Maria Beatrice Di Bisceglie ${ }^{1}$, Laura Fatone ${ }^{1}$, Gianfranco Favia ${ }^{1}$ \\ 1. Interdisciplinary Department of Medicine, University of Bari, Italy; \\ 2. Research Assistant and Professor, Department of Basic Medical Sciences, Neurosciences and Sensory Organs, University of Bari, Italy.
}

$\triangle$ Corresponding author: Prof. Vito Crincoli, Interdisciplinary Department of Medicine, Piazza Giulio Cesare 11, 70124, Bari, Italy. Phone: 00390805478051 Fax: 00390805478743 E-mail: vito.crincoli@uniba.it

(C) 2015 Ivyspring International Publisher. Reproduction is permitted for personal, noncommercial use, provided that the article is in whole, unmodified, and properly cited. See http://ivyspring.com/terms for terms and conditions.

Received: 2014.12.09; Accepted: 2015.03.24; Published: 2015.04.26

\begin{abstract}
AIMS: Psoriasis is a chronic, remitting and relapsing inflammatory disorder, involving the skin, nails, scalp and mucous membranes, that impairs patients' quality of life to varying degrees. Psoriatic arthritis is a chronic seronegative, inflammatory arthritis, usually preceded by psoriasis. Temporomandibular disorders is a generic term referred to clinical conditions involving the jaw muscles and temporomandibular joint. The aim of this study was to assess symptoms and signs of temporomandibular disorders in psoriasis patients with and without psoriatic arthritis.

METHODS: The study group included 112 patients ( 56 men, 56 women; median age $49.7 \pm 12$ years) with psoriasis, 25 of them were affected by psoriatic arthritis. A group of 112 subjects without psoriasis ( 56 men, 56 women; median age $47.7 \pm 17$ years) served as controls. Signs and symptoms of temporomandibular disorders were evaluated according to the standardized Research Diagnostic Criteria for Temporomandibular Disorders. Psoriasis patients were subgrouped according to the presence/absence of psoriatic arthritis and by gender, to assess the prevalence of traditional symptoms and signs of temporomandibular disorders.

RESULTS: Patients with psoriasis, and to an even greater extent those with psoriatic arthritis, were more frequently affected by symptoms and signs of temporomandibular disorders, including an internal temporomandibular joint opening derangement than healthy subjects. A statistically significant increase in symptoms of temporomandibular disorders, in opening derangement, bruxism and sounds of temporomandibular joint was found in patients with psoriatic arthritis as compared with psoriasis patients without arthritis and controls.
\end{abstract}

CONCLUSIONS: psoriasis seems to play a role in temporomandibular joint disorders, causing an increase in orofacial pain and an altered chewing function.

Key words: psoriasis, psoriatic arthritis, temporomandibular disorders. RDC/TMD.

\section{Introduction}

Psoriasis is a chronic, remitting and relapsing inflammatory disorder, involving the skin, nails, scalp and mucous membranes, that impairs patients' quality of life to varying degrees [1-3]. This disease affects $1-3 \%$ of the world population [3] and in approximately one-third of patients heredity is a factor $[4,5]$.
Local trauma, preceding mild inflammatory disease, and psychosomatic factors seem to play an important role in the incidence of psoriasis, while gender and age are considered irrelevant $[1,2,4,5]$.

Symptoms such as itching of the skin, pain and burning sensations of the oral mucosa, especially 
when associated with stress, may alter the chewing function, due to muscular hyperactivity [9].

Signs present in the oral environment may involve the soft tissues and include a geographic tongue, fissured tongue, as well as gingival and/or mucosal lesions [3].

The relationship between psoriasis and arthritis was first observed by Alibert in 1818, and identified as a clinical entity by Bazin in 1860 [6, 7]. Psoriatic arthritis (PsA) is a chronic seronegative, inflammatory arthritis, usually preceded by psoriasis [8] and can directly affect the hard tissues, such as the temporomandibular joint (TMJ) [10, 11].

Temporomandibular disorder (TMD) is a generic term referred to clinical conditions involving the jaw muscles and TMJ $[12,13]$. Such disorders are related to stress, age, gender, malocclusion and other systemic factors [14]. It is estimated that about one third of adults suffer from TMD symptoms (TMDs) [15].

The classification of TMDs includes three subcategories: derangements of the condyle-disc complex, structural incompatibility of the articular surfaces and inflammatory disorders [16].

The aim of this study was to assess symptoms and signs of TMD in patients with psoriasis and PsA, as compared with a healthy control group, and to investigate the relationship between TMD and psoriasis.

\section{Materials and Methods}

This study was conducted from January to December 2014 at the School of Dentistry, University of Bari, Italy, in accordance with the provisions of the Declaration of Helsinki. Institutional human experimentation panel approval and informed consent from each human subject were obtained.

Over 18 years of age, gender and European ethnic origin were inclusion criteria. Exclusion criteria were: previous history of acute traumatic injury, dental emergencies, drug abuse, head and neck neoplasia, vascular disease, visual, auditory, or motor impairments [17-19] and neurological disorders. In addition, patients receiving or having undergone orthodontic treatment or orthognatic surgery were not selected.

One hundred twelve patients (56 men, 56 women) with psoriasis were enrolled.

Twenty-five of the recruited patients were affected by PsA, according to the Moll \& Wright criteria [20]. A group of 112 patients without psoriasis (56 men, 56 women), randomly selected among those presenting at the Dental Clinic, served as control group. Patients age ranged between 18 and 76 years, with a median age of 49.7 ( $\mathrm{SD} \pm 12$ ) years in the psoriatic group, and 47.7 years $(\mathrm{SD} \pm 17)$ in the controls.
The examination for TMD signs and symptoms was based on the standardized Research Diagnostic Criteria for Temporomandibular Disorders (RDC/TMD) [18]. TMD were assessed by means of a questionnaire and a thorough clinical examination. Symptoms and signs were recorded by a single experienced practitioner, who was blind to group allocation.

Signs and symptoms in the whole psoriatic population (with and without PsA) were compared with those in the control group, and a separate comparison was also made of the female subgroups. Within the psoriatic group, a subgroup denoted "psoriasis with PsA", composed of 25 individuals, accounted for $22.3 \%$ of the psoriatic sample. This group was compared with two different subgroups, matched for number of individuals, age and gender: psoriasis patients without PsA and healthy controls. Comparison was also made between the psoriatic population without PsA and the healthy controls.

\section{Symptoms}

\section{Patients history}

All patients' complaints (TMDs) were recorded by means of a questionnaire enquiring about: muscle pain, ranging from slight tenderness to extreme pain (VAS scale), tenderness or stiffness in the neck and upper shoulders, muscle pain during use of the jaw (chewing, etc.), tenderness or pain in the joint area (arthralgia), difficulty in opening the mouth, a feeling that the jaw was stuck or locked, headaches [19].

Other, less common symptoms, such as dizziness, earache and ringing in the ears (tinnitis) were also investigated [21].

All these data were collected as categorical ones (presence or absence of TMDs).

\section{Clinical examination}

The most common symptom, the myofascial pain (MP), was accurately evaluated.

While healthy muscles do not elicit sensations of tenderness or pain when palpated, an ache may be elicited by compression of damaged muscle tissue. These data were collected as categorical (presence or absence of tenderness) after digital palpation of the jaw muscles. Palpation was performed mainly by the palmar surface of the thumb and of the index finger, applying soft but firm pressure to the muscle.

\section{Signs}

\section{TMJ sounds (TMJs)}

Clicking is considered as a single, loud joint sound of short duration, similar to a pop, while crepitation as multiple, rough, gravel-like sounds described as grating [19]. 


\section{Bruxism (BRUX)}

It is a stereotypical mandibular movement characterized by grinding, clenching and gnashing of the teeth; it can occur during sleep or when awake [22]. Central neurological circuits, particularly dopaminergic neurotransmission, seem to be the main cause of bruxism [23, 24]. This phenomenon becomes pathological when associated to nonadaptive processes, causing muscle or joint pain. Therefore, patients are considered to suffer from bruxism only when:

(i) myalgia is associated with the parafunction. The muscular pain is caused by prolonged vasoconstriction, and there is accumulation of catabolites in the muscle tissue [25].

(ii) the development of wear facets, i.e. bright flat areas that alter the natural occlusal form of the teeth, is detected.

\section{Opening derangement (OD)}

In the healthy masticatory system, the path taken by the mandible midline in maximum opening is straight. Alterations of the opening trajectory were classified as:

(i) deviation: any shift of the jaw midline during opening that disappears with continued opening (a return to midline);

(ii) deflection: any shift of the midline to one side that becomes greater with opening and does not disappear at maximum opening [26].

\section{Restricted movements (RM)}

(i) Reduced opening: in a healthy system, the mouth opens by between 53 and $58 \mathrm{~mm}$. Taking into account overbite [27], a restricted mandibular opening is considered to be any distance of $<40 \mathrm{~mm}$.

(ii) lateral excursions, measured from midline to midline, were recorded as distance lower than $8 \mathrm{~mm}$.

(iii) a mandibular protrusion (mean values ranging between 7 and $10 \mathrm{~mm}$ ) was recorded when $<7 \mathrm{~mm}$ [28].

\section{Statistical Analysis}

The prevalence of signs and symptoms was analyzed in all the groups described above. Categorical data were expressed as number and percentage and compared using chi-squared $\left(\chi^{2}\right)$ test with Yates' correction or Fisher's exact test. In all comparisons a $p$ value of $\leq 0.05$ was considered statistically significant. (Epilnfo ver 3.5.3).

\section{Results}

\section{Psoriatic versus healthy group (Fig. 1A)}

\section{Symptoms}

The assessment of TMDs showed that $68.8 \%$ of the patients with psoriasis and $24.1 \%$ of the healthy controls suffered from one or more symptoms. Large statistically significant differences were found between the two groups ( $p$ value $<0.001)$.

In the psoriasis group, tenderness or stiffness in the neck and shoulders, muscle pain on chewing and the sensation of a stuck or locked jaw were the most commonly reported symptoms. In the control group, tenderness or stiffness in the neck and shoulders was the major complaint.

MP evoked by palpation was detected in 58\% psoriatic patients and $56.3 \%$ of controls, revealing no statistically significant difference between the two groups ( $p$ value $>0.05)$.

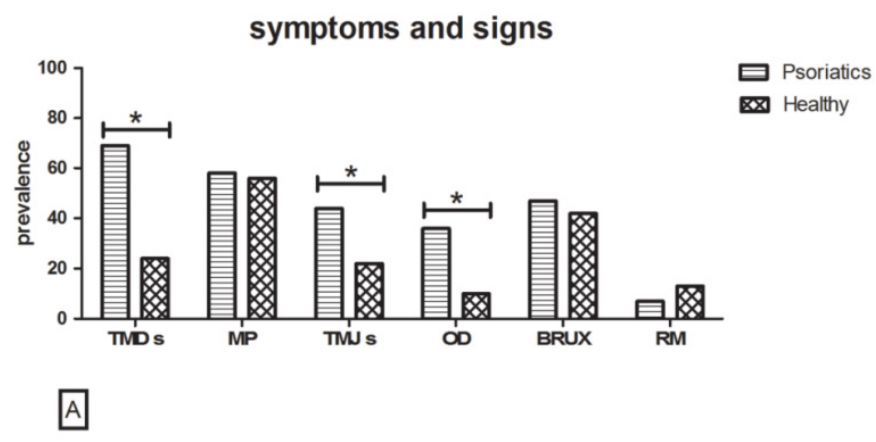

symptoms and signs (female subgroups)

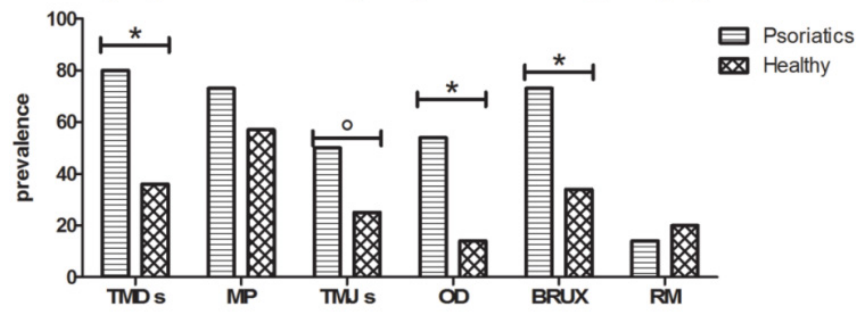

B

Figure 1. [A] Symptoms and signs in Psoriatic versus Healthy group. Data reveal a higher prevalence of TMDs, TMJs and OD in the Psoriatic group $\left(X^{2}\right.$ test with Yates' correction, $\left.{ }^{*} p<0.001 n=112\right)$. The remaining evaluations show no statistically significant differences. [B] Symptoms and signs in Psoriatic versus Healthy in female subgroup. Results indicate a higher prevalence of TMDs, TMJs, OD once again. In addition, in the female population of psoriasis patients, BRUX represents a very recurring sign ( $X^{2}$ test with Yates' correction ${ }^{*} p<0.01,{ }^{\circ} p=0.03 n=56$ ).

\section{Signs}

At the clinical examination, TMJs and OD were more frequent in the psoriasis group than in the controls. In particular, $44.3 \%$ of psoriasis patients showed TMJs versus $22.3 \%$ of controls ( $p$ value $<0.001$ ): clicking was recorded in $33 \%$ of the psoriasis group (with and without PsA) and $15.2 \%$ of controls, with a highly significant difference $(p$ value $=0.003)$ and crepitation 
in $11.3 \%$ of psoriasis patients and in $7.1 \%$ of controls ( $p$ value $>0.05$ ).

Positive findings for OD were recorded in 35.7\% of psoriasis patients versus $9.8 \%$ of controls, showing statistically significant differences $(p$ value $<0.001)$ between the two groups.

Evidence of bruxism was observed in $47.3 \%$ of patients with psoriasis and $42 \%$ of controls, with no significant difference between the two groups ( $p$ value $>0.05$ ).

Finally, the prevalence of RM was higher in controls $(12.5 \%)$ than in psoriasis patients $(7.1 \%)$, although this difference was not statistically significant ( $p$ value $>0.05$ ).

\section{Psoriasis patients versus healthy controls in the female subgroup (Fig. IB)}

\section{Symptoms}

When performing the same assessments limited to the female population, quite similar results were obtained. TMDs were referred by $80.4 \%$ of psoriasis patients versus $35.7 \%$ of healthy female controls $(p$ value $<0.001$ ), whereas once again, MP evoked by palpation revealed no statistically significant differences between the two groups, although $73.2 \%$ of psoriasis patients were positive to the test versus $51.7 \%$ of controls ( $p$ value $>0.05$ ).

\section{Signs}

At the clinical examination, TMJs and OD continued to be more frequent in the female psoriasis group than the controls. In particular, $50 \%$ of psoriasis patients versus $25.4 \%$ of controls showed TMJs ( $P$ value $=0.03$ ). Analyzing the TMJs, $34.6 \%$ of female psoriasis patients and $14.5 \%$ of controls had clicking ( $p$ value $=0.03$ ) while $15.4 \%$ of psoriasis patients and $10.9 \%$ of controls were positive for crepitation, with no statistically significant difference. In the female subgroups, $53.6 \%$ of psoriasis patients versus $14.3 \%$ of controls were positive for OD ( $p$ value $<0.001)$.

Evidence of bruxism was observed in $73.2 \%$ of female patients with psoriasis versus $33.9 \%$ of controls, showing highly significant differences between the two groups ( $p$ value $<0.001$ ).

Finally, there was no difference ( $p$ value $>0.05)$ in the prevalence of RM between the female controls $(19.6 \%)$ and psoriasis patients $(14.3 \%)$.

\section{PsA versus healthy controls (Fig. 2A)}

\section{Symptoms}

Comparing the same symptoms between psoriasis patients with PsA and their healthy controls, TMDs were recorded in $80 \%$ of psoriasis patients with PsA and $28 \%$ of the controls ( $p$ value $=0.0007$ ), show- ing a highly statistically significant difference. Even if there were no statistically significant differences in MP between the two groups, $72 \%$ of psoriasis patients with PsA suffered pain during palpation of the jaw versus $56 \%$ of the controls ( $p$ value $>0.05$ ).

\section{Signs}

At the clinical examination, TMJs, OD, and bruxism were more frequent between psoriasis patients with PsA than controls, this difference being statistically significant. In detail, TMJs were detected in $62.7 \%$ of psoriasis patients with PsA versus $20 \%$ of controls ( $p$ value $=0.02$ ). Clicking was more frequent in the PsA subgroup than in healthy controls $(40.7 \%$ versus $16 \%$ ), with a statistically significant difference ( $p$ value $=0.04$ ). Even if crepitation was more frequent in the PsA subgroup (22.2\%) than in the controls (4\%), no statistically significant differences were found. OD were observed in $56 \%$ of psoriasis patients with PsA and in $12 \%$ of the healthy controls ( $p$ value $=0.003$ ).

Bruxism was present in $68 \%$ of patients in the psoriasis group with PsA and in $40 \%$ of controls $(p$ value $=0.044$ ).

The only sign that did not show any difference between the two groups was RM, observed in $16 \%$ of subjects in both subgroups. ( $p$ value $>0.05$ ).

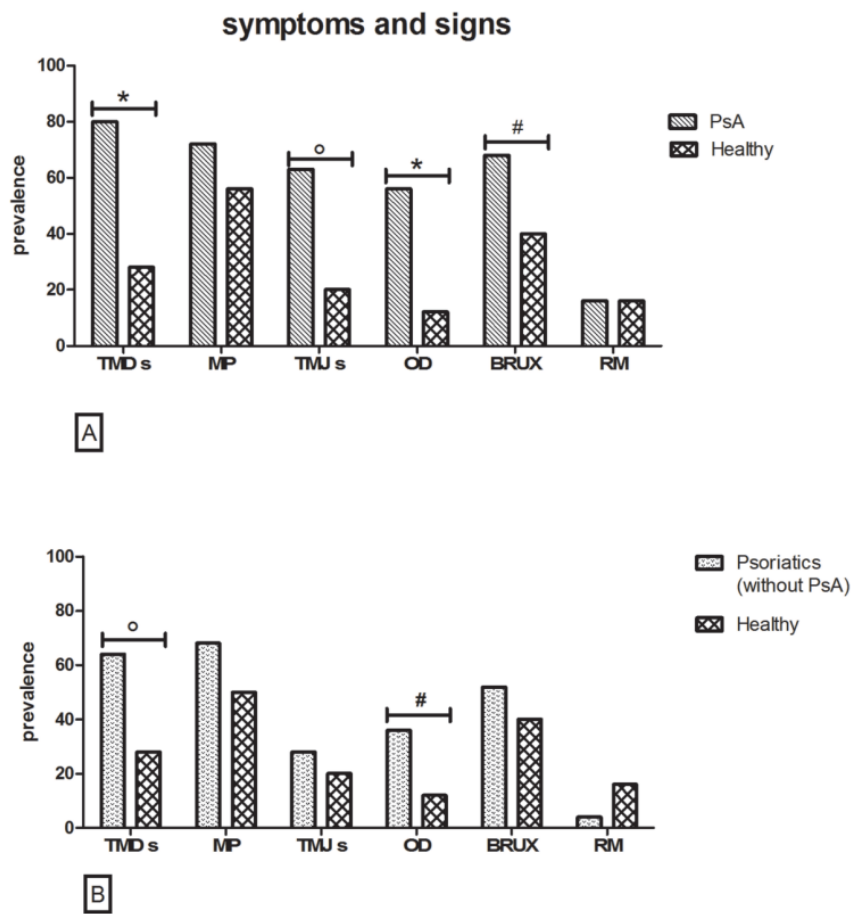

Figure 2. [A] Symptoms and signs in PsA versus Healthy subgroup. As regard to TMDs, TMJs and OD ( $X^{2}$ test with Yates' correction $* p<0.01$, ${ }^{\circ} p=0.02 n=25$ ), show huge statistically significant differences. The prevalence of BRUX appears higher in PsA (Fisher exact test, $\# p<0.05 n=25$ ). [B] Symptoms and signs in Psoriatic without PsA versus Healthy subgroup. Diagram reveals a higher prevalence of TMDs ( $X^{2}$ test with Yates' correction, ${ }^{\circ} p=0.02 n=25$ ) and OD (Fisher exact test, $\# p<0.05 n=25$ ) in the Psoriasis patients without PsA. 


\section{Psoriasis patients without PsA versus healthy controls (Fig. 2B)}

\section{Symptoms}

When performing the same assessments within the psoriatic population without PsA compared to the healthy controls, quite similar results were obtained. In particular, $64 \%$ of psoriasis patients were affected by TMDs versus $28 \%$ of healthy controls ( $p$ value $<0.02$ ), whereas no statistically significant differences between the two groups ( $p$ value $>0.05$ ) were revealed for MP evoked by palpation, even if $68 \%$ of psoriasis patients versus $56 \%$ of controls were positive to palpation.

\section{Signs}

Statistically significant differences were observed only for OD, present in $36 \%$ of psoriasis patients without PsA and in $12 \%$ healthy controls $(p$ value $=0.048$ ).

The other signs showed no statistically significant differences between the two groups. In particular, $52 \%$ of psoriasis patients versus $40 \%$ of controls were positive for bruxism ( $p$ value $>0.05$ ), while $4 \%$ of psoriasis patients versus $16 \%$ of controls displayed RM and $28 \%$ of psoriasis patients versus $20 \%$ of controls showed TMJs ( $p$ value $>0.05$ ). Finally, clicking was detected in $28 \%$ of psoriasis patients without PsA and in $16 \%$ of controls ( $p$ value $>0.05)$ and crepitation was recorded only in controls $(4 \%)$.

\section{PsA versus psoriasis without PsA subgroups (Fig. 3)}

\section{Symptoms}

No statistically significant differences ( $p$ value $>0.05$ ) between the two subgroups were found as regards TMDs $(80 \%$ of PsA and $64 \%$ of psoriasis patients without PsA) and MP (72\% of PsA versus $68 \%$ of psoriasis patients without PsA).

\section{Signs}

TMJs and OD were more frequent in the psoriasis with PsA subgroup than in the psoriasis without PsA subgroup. In particular, $62.7 \%$ of psoriasis patients with PsA versus $28 \%$ of patients without PsA showed TMJs, this difference being statistically significant ( $p$ value $=0.042$ ). In particular, clicking was detected in $40.7 \%$ of the PsA subgroup and $28 \%$ of the psoriasis without PsA subgroup, with no statistically significant difference; on the other hand, crepitation was more frequent in the PsA subgroup $(22.2 \%)$ than in the psoriasis without PsA subgroup $(0 \%)$ ( $p$ val$\mathrm{ue}=0.01$ ). Even if OD were more frequent in the subgroup with psoriasis and PsA (56\%) than the subgroup without PsA (36\%), there was no statistically significant difference between the two subgroups ( $p$ value $>0.05$ ).

Evidence of bruxism was observed in $68 \%$ of psoriasis patients with PsA and $52 \%$ of patients without PsA, with no significant difference between the two groups ( $p$ value $>0.05$ ).

Finally, the prevalence of RM in the psoriasis patients $(16 \%)$ as compared to the psoriasis with PsA patients $(4 \%)$ did not reveal any differences between the two groups ( $p$ value $>0.05)$.

\section{symptoms and signs}

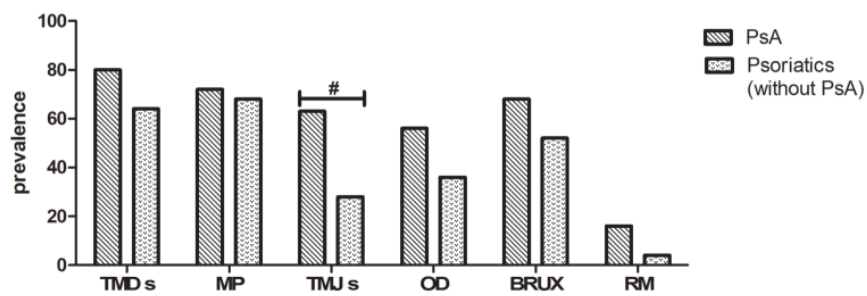

Figure 3. Symptoms and signs in PsA versus Psoriatic without PsA subgroup. The two subgroups differ only in a higher prevalence of TMJs in PsA patients. (Fisher exact test, $\# p<0.05, \mathrm{n}=25$ ).

\section{Discussion}

Several studies have reported that the prevalence of TMJ symptoms and signs varies widely in the general population [10, 24, 26, 29-31], but data are lacking on the prevalence of TMD in psoriasis and in PsA.

According to Zhou et al., inflammatory TMJ lesions in psoriasis patients do not seem to be a common condition, being reported in less than $5 \%$ of patients [3].

Using a postal questionnaire, Könönen [32] selected a PsA subgroup within a psoriatic group, thus not adopting objective criteria, but patients' self-reported general joint symptoms. In another study, [17] the same Author selected PsA patients according to the Moll \& Wright criteria, as did Dervis [5]. Through a clinical investigation of TMD and radiographic features in patients with PsA, Könönen reported that involvement of the TMJ might be common [17, 32-34]. In a study by Wenneberg, radiographically observed erosive changes were significantly more frequent in subjects with PsA $(38 \%)$ than in controls (12\%) [30].

The results of the present investigation showed that TMDs were remarkably prevalent among the psoriasis patients as compared with healthy controls. The prevalence rate of TMDs was constantly higher in the psoriatic group and subgroups (females, psoriasis patients with or without PsA) than in the controls, these differences being highly statistically significant.

In detail, TMDs were found in $68.8 \%$ of the 
whole sample of psoriasis patients (with and without PsA), showing an approximately three-fold higher frequency than in the healthy controls $(24.1 \%)$.

TMDs were present in $64 \%$ of psoriatic patients without PsA. This result differs from that of a similar study by Dervis et al. [5], where only $29.4 \%$ of patients with psoriasis without arthritis complained of TMDs, and from the $28 \%$ found by Könönen [32].

Finally, the prevalence of TMDs was higher in the PsA subgroup (80\%), probably because arthritis affects the conditions of the TMJ [31], but with no statistically significant differences as compared to psoriasis patients without PsA. Once again, these data differ greatly from those of Dervis (35\%) [5] and Könönen (48\%) [17].

Tenderness or stiffness were found in the neck and shoulders, muscle pain on chewing and a sensation of the jaw being stuck or locked as the most commonly reported symptoms.

Similar findings were obtained for tenderness evoked by palpation of the jaw muscles in both groups, 58\% in the psoriatic groups (with and without PsA) and $56.3 \%$ in the control group. However, analyzing the single subgroups, MP was more frequently observed in the psoriatic subgroups with $(72 \%)$ or without PsA (68\%) than in their controls (56\%), although no statistically significant differences were found.

In a study carried exclusively on PsA patients, Könönen obtained similar data: MP was reported by $62 \%$ patients in the PsA group and in $50 \%$ of the controls [17]. Dervis found a lower prevalence: MP was detected in $14.7 \%$ in the psoriatic group without PsA versus $22 \%$ of the control group, and in $40 \%$ of the PsA group versus $10 \%$ of the controls [5]. Such a difference between these studies could be explained by the absence of a standardized pressure during manual palpation performed by several operators.

Taking into account gender, TMDs were more frequent in all the female subgroups than in the whole psoriasis patients sample and controls. Although our percentage values are higher, the ratio between males and females is in accordance with literature [35]. This higher prevalence of symptoms in females than males could be explained by behavioral, psychosocial, hormonal (estrogen receptors in the TMJ modulating laxity of the ligaments or in the limbic system increasing vigilance in relation to pain stimuli) and constitutional differences [20].

As regards TMJs, $44.3 \%$ of psoriasis patients (with and without PsA) and $22.3 \%$ of controls were positive, with an important statistical significance.

Moreover, extrapolating data about TMJs in the psoriatic population without PsA $(28 \%)$, and comparing them with those of the psoriatic population with PsA (62.7\%), the ratio is more than double, quite similar to Könönen's one. He reported TMJs in $22 \%$ of psoriatic and $41 \%$ of PsA patients [13]. These data seem to confirm that psoriasis, and even more PsA, may play an important role in the etiopathogenesis of intracapsular disorders.

Within TMJs, a distinction of clicking and crepitation has been made (Fig. 4) [5]. Clicking is a single loud joint sound of short duration, prompting a disc derangement. Crepitation is suggestive of structural osseous changes in the joint, increasing with age, caused by PsA or by osteoarthrosis [17]. Both clicking and crepitation were considerably more frequent ( $40.5 \%$ and $22.2 \%$, respectively) in the PsA subgroup than in controls (16\% and $4 \%$, respectively) and the psoriasis (only clicking $28 \%$ ) subgroups.

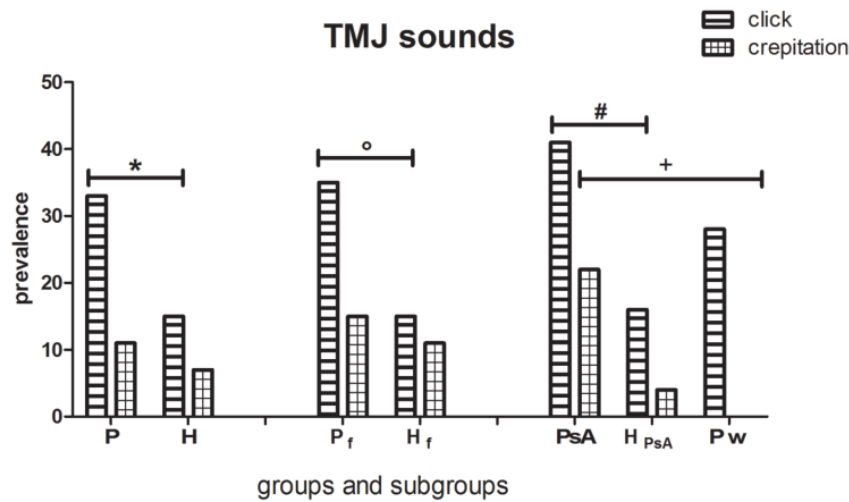

Figure 4. Click and Crepitation in the groups and subgroups evaluated. Data reveal a higher prevalence of clicking in the P (Psoriatic group ), Pf (Psoriatic female ) and PsA (Psoriatic Arthritis) subgroups compared to their healthy controls $\mathrm{H}$ (whole healthy controls), $\mathrm{H}_{\mathrm{f}}$ (healthy female controls) and $\mathrm{H}_{P_{s A}}$ (healthy controls matched for number, age and gender with PsA patients) respectively ( $X{ }^{2}$ test with Yates' correction ${ }^{*} p<0.001 n=112$; ${ }^{\circ} p=0.01 n=56$; Fisher exact test, $\# p<0.05 n=25)$. PsA patients also show a higher prevalence of crepitation (Fisher exact test, ${ }^{+} \mathrm{P}<0.02 \mathrm{n}=25$ ) when compared to $\mathrm{Pw}$ (psoriatic subgroup without PsA).

Könönen found clicking in 39\% of PsA patients (vs $27 \%$ of controls) and crepitation in $58 \%$ of PsA patients (vs 34\% of controls) [17]. Dervis did not find any statistical significant difference between psoriatic patients and controls: clicking was found in $25 \%$ of psoriatic patients and $23.5 \%$ of controls, crepitation in $4.4 \%$ of the psoriatic group and $5.8 \%$ of controls. He performed a similar assessment for clicking in the PsA group (15\%) and the controls (25\%). Crepitation was higher in the PsA group (30\%) than the controls (5\%) [5], these data being similar to those obtained in the present study $(22.2 \%$ in the PsA subgroup and $4 \%$ of the controls) but lower than Könönen's findings (58\%) [17]. Frank's study [37] reported that crepitation is most commonly associated with osteoarthritic changes of the articular surfaces of the joint and that it is a late phase of involvement of the masticatory system with structural changes in the joints without pain. 
Specifically, when PsA affects the TMJ and is not successfully stopped in the early degenerative process, patients may undergo invasive joint remodeling that irreversibly changes the physiologic TMJ anatomy and dynamics [31]. Clinically, TMJ involvement by PsA causes sounds and gradually altered TMJ movements $[10,17]$.

Bruxism was observed in a similar percentage both in psoriasis patients and the control group ( $47.3 \%$ and $42 \%$, respectively), but, analyzing the single subgroups, higher frequencies were found in the psoriatic subgroups with or without PsA than in controls. The parafunction was present in $68 \%$ of patients in the PsA subgroup, 52\% of psoriasis patients and $40 \%$ of controls. These differences, even if statistically significant only between the PsA group and the relative controls, could be explained by an increased stress in patients with psoriasis with or without PsA than in controls [37]. Recent works have shown that a third of patients with psoriasis suffered from pathological worry and anxiety and that psychological interpersonal difficulties have an impact on all aspects of the patient's daily life [38, 39].

Dervis observed parafunctional jaw habits in $16.1 \%$ of psoriatic patients without PsA and in $15 \%$ of PsA patients [5]. Könönen assessed nocturnal and diurnal bruxism: in the PsA group, nocturnal bruxism was found in $25 \%$ and diurnal in $34 \%$ of patients, in the psoriatic group nocturnal bruxism was estimated to be $24 \%$ and diurnal $31 \%$ [32]. The present study showed a higher prevalence of this symptom.

An alteration of the physiological relationship between the condyle and disc in the glenoid fossa causes an impairment of the mandible opening path. OD was found in $35.7 \%$ of the psoriatic group and $9.8 \%$ of the controls, with an important statistical difference. In detail, this kinematic disorder was present in $56 \%$ of PsA patients, in $36 \%$ of psoriatic patients without PsA and in $12 \%$ of the relative controls. These percentages differ from those recorded by Dervis, where OD found in the psoriatic group and in their controls were equal to $20 \%$ and $15 \%$ respectively, while in PsA patients and their controls corresponded to $17.6 \%$ and $13.2 \%$ [5]. By matching data related to OD with those of TMJs in the same subgroups, it is possible to observe a linear correspondence.

Analyzing the reduction in the width of mandibular movement (RM), contrary to expectations, a slightly higher prevalence of this sign was observed in the control group than in the psoriatic group $(12.5 \%$ versus $7.1 \%$ ). About subgroups, RM were found in $16 \%$ of the PsA subgroup, in $4 \%$ of the psoriatic subgroup and in $16 \%$ of the controls, with no statistically significant differences. A similar value was recorded by Könönen using the postal questionnaire $(6 \%$ in the psoriatic group and $13 \%$ in the PsA group) [32], while Dervis found values of $2.9 \%$ in the psoriatic group and $0 \%$ in the PsA group (0\%) [5].

\section{Conclusions}

In this observational study, a statistically significant increase of TMD symptoms (TMDs) was consistently detected in patients with psoriasis and, even more, with PsA, as compared to a sample of healthy subjects. However, muscular pain (MP) evoked by palpation was quite similar in the two groups and all the subgroups. TMJ sounds (TMJs) and Opening Derangement (OD) were found to be more frequent and severe in patients with psoriasis and PsA than in the healthy subjects, this result being highly significant. Bruxism was remarkably increased only in the female psoriatic and in the PsA subgroups. Finally, no notable difference was observed as regards to a restricted mandibular opening.

Therefore, in addition to dermatological and rheumatological implications, psoriasis seems to play a role in TMJ disorders, causing an increase in orofacial pain and an altered chewing function.

\section{Abbreviations}

PsA: psoriatic arthritis; TMD: temporomandibular disorders; TMJ: temporomandibular joint; RDC/TMD: research diagnostic criteria for temporomandibular disorders; TMDs: symptoms of temporomandibular disorders; OD: opening derangement; BRUX: bruxism; TMJs: sounds of temporomandibular joint; VAS: visual analogue scale; MP: myofascial pain; RM: restricted movements.

\section{Competing Interests}

The authors have declared that no competing interest exists.

\section{References}

1. Ingram JT. Approach to psoriasis. Br Med J. 1953; 12:591-4.

2. Baker H. Epidemiological aspects of psoriasis and arthritis. Br J Dermatol. 1966; 78:249-61.

3. Zhu JF, Kaminski MJ, Pulitzer DR, Hu J, Thomas HF. Psoriasis: pathophysiology and oral manifestations. Oral Dis. 1996; 2:135-44.

4. Watson W, Cann HM, Farber E, Nall ML. The genetics of psoriasis. Arch Dermatol. 1972; 105:197-207.

5. Dervis E, Dervis E. The prevalence of temporomandibular disorders in patients with psoriasis with or without psoriatic arthritis. J Oral Rehabil. 2005; 32:786-93.

6. Laskin DM. Ethiology of the pain-disfunction syndrome. J Am Dent Assoc. 1969; 79:147-53.

7. Gladman D, Shucket M, Russell J, Thorne J, Schacthter R. Psoriatic arthritis (PSA)-an analysis of 220 patients. Q J Med. 1987; 62:127-41.

8. Brockbank J, Gladman D. Diagnosis and management of psoriatic arthritis. Drugs. 2002; 62:2447-57.

9. Gladman DD. Psoriatic arthritis. Rheum Dis Clin North Am. 1998; 24:829-44.

10. Lundberg M, Ericsson S. Changes in the temporomandibular joint in psoriasis arthropathica. Acta Derm Venereol. 1967; 47:354-60.

11. Könönen M. Craniomandibular disorders in psoriasis. Community Dent Oral Epidemiol. 1987; 15:108-12.

12. McNeill C. Management of temporomandibular disorders: Concepts and controversies. J Prosthet Dent. 1997; 77:510-22. 
13. Okeson JP, de Leeuw R. Differential diagnosis of temporomandibular disorders and other orofacial pain disorders. Dent Clin North Am. 2011; 55:105-20.

14. Oral K, Bal Küçük B, Ebeoğlu B, Dinçer S. Etiology of temporomandibular disorder pain. Agri.2009; 21:89-94.

15. Buescher JJ. Temporomandibular joint disorders. Am Fam Physician. 2007; 76:1477-82.

16. Okeson JP. Signs and symptoms of temporomandibular disorders. In: Okeson JP, ed. Management of temporomandibular disorders and occlusion. 6th ed. St Louis: Mosby Elsevier; 2008:164-215.

17. Könönen M. Clinical signs of craniomandibular disorders patients with psoriatic arthritis. Scand J Dent Res. 1987; 95:340-6.

18. Dworkin SF, LeResche L. Research diagnostic criteria for temporomandibular disorders: review, criteria, examinations and specifications, critique. J Craniomandib Disord. 1992; 6:301-55.

19. Tecco S, Crincoli V, Di Bisceglie B, Saccucci M, Macrì M, Polimeni A, Festa F. Signs and symptoms of temporomandibular joint disorders in Caucasian children and adolescents. Cranio. 2011; 29:71-9.

20. Helliwell $\mathrm{P}$, Taylor $\mathrm{W}$. Classification diagnostic criteria for psoriatic arthritis. Ann Rheum Dis. 2005; 64 (Suppl 2):ii3-ii8.

21. Buergers R, Kleinjung T, Behr M, Vielsmeier V. Is there a link between tinnitus and temporomandibular disorders? J Prosthet Dent. 2014; 111·222-7.

22. Lavigne GJ, Khoury S, Abe S, Yamaguchi T, Raphael K. Bruxism physiology and pathology: an overview for clinicians. J Oral Rehabil. 2008; 35:476-94.

23. Mehulić K, Gospić RK, Dundjer A, Skrinjarić T, Stefancić S, Vojvodić D, Perinić M. Optoelectronic pantography diagnostics of temporomandibular disorders in patients with bruxism. Coll Antropol. 2009; 33:849-56.

24. Lavigne GJ, Kato T, Kolta A, Sessle BJ. Neurobiological mechanisms involved in sleep bruxism. Crit Rev Oral Biol Med. 2003; 14:30-46.

25. Bell WE. Orofacial pains: differential diagnosis. 2nd ed. Chicago: Year Book Medical Publishers, Inc; 1979.

26. Sener S, Akgunlu F. Correlation between the Condyle Position and Intra-Extraarticular Clinical Findings of Temporomandibular Dysfunction. Eur J Dent. 2011; 5:354-60.

27. Agerberg G. et al. Maximal mandibular movements in young men and women. Sven Tandlak Tidskr. 1974; 67:81-100.

28. Solberg W. Occlusion-related pathosis and its clinical evaluation. Clinical dentistry. New York: Harper \& Row Publishers; 1976: 1-29.

29. Moll JM, Wright V. Psoriatic arthritis. Semin Arthritis Rheum. 1973; 3:55-78.

30. Wenneberg B, Könönen M, Kallenberg A. Radiographic changes in the temporomandibular joint of patients with rheumatoid arthritis, psoriatic, arthritis, and ankylosing spondylitis. J Craniomandib Disord. 1990; 4:35-9.

31. Lamazza L, Guerra F, Pezza M, Messina AM, Galluccio A, Spink M, De Biase A. The use of etanercept as a non-surgical treatment for temporomandibular joint psoriatic arthritis: a case report. Aust Dent J. 2009; 54:161-5.

32. Könönen M. Craniomandibular disorders in psoriasis. Community Dent Oral Epidemiol. 1987; 15:108-12.

33. Könönen M. Craniomandibular disorders in psoriatic arthritis. Correlations between subjective symptoms, clinical signs, and radiographic changes. Acta Odontol Scand. 1986; 44:369-75.

34. Könönen M. Subjective symptoms form the stomatognathic system in patients with psoriatic arthritis. Acta Odontol Scand. 1986; 44:377-83.

35. Warren MP Fried JL. Temporomandibular Disorders and Hormones in Women. Cells Tissues Organs. 2001; 169:187-92.

36. Franks AS. Temporomandibular joint in adult rheumatoid arthritis. A comparative evaluation of 100 cases. Ann Rheum Dis. 1969; 28:139-45.

37. Gupta MA, Gupta AK, Ellis CN, Voorhees JJ. Some psychosomatic aspects of psoriasis. Adv Dermatol. 1990; 5:21-30.

38. Langley RG, Krueger GG, Griffiths CE. Psoriasis: epidemiology, clinical features, and quality of life. Ann Rheum Dis. 2005; 64(Suppl 2):ii18-ii23.

39. Fortune DG, Main CJ, O'Sullivan TM, Griffiths CE. Assessing illness related stress in psoriasis: the psychometric properties of the Psoriasis Life Stress Inventory. J Psychosom Res. 1997; 42:467-75. 